UCRL-JC-118789

PREPRINT

\title{
Experimental Status of the AGS Relativistic Heavy Ion Program
}

\author{
T. Craig Sangster
}

This paper was prepared for submittal to the Proceedings of the 27th International Conference on High Energy Physics Glasgow, Scotland July 20-27, 1994

October 1994

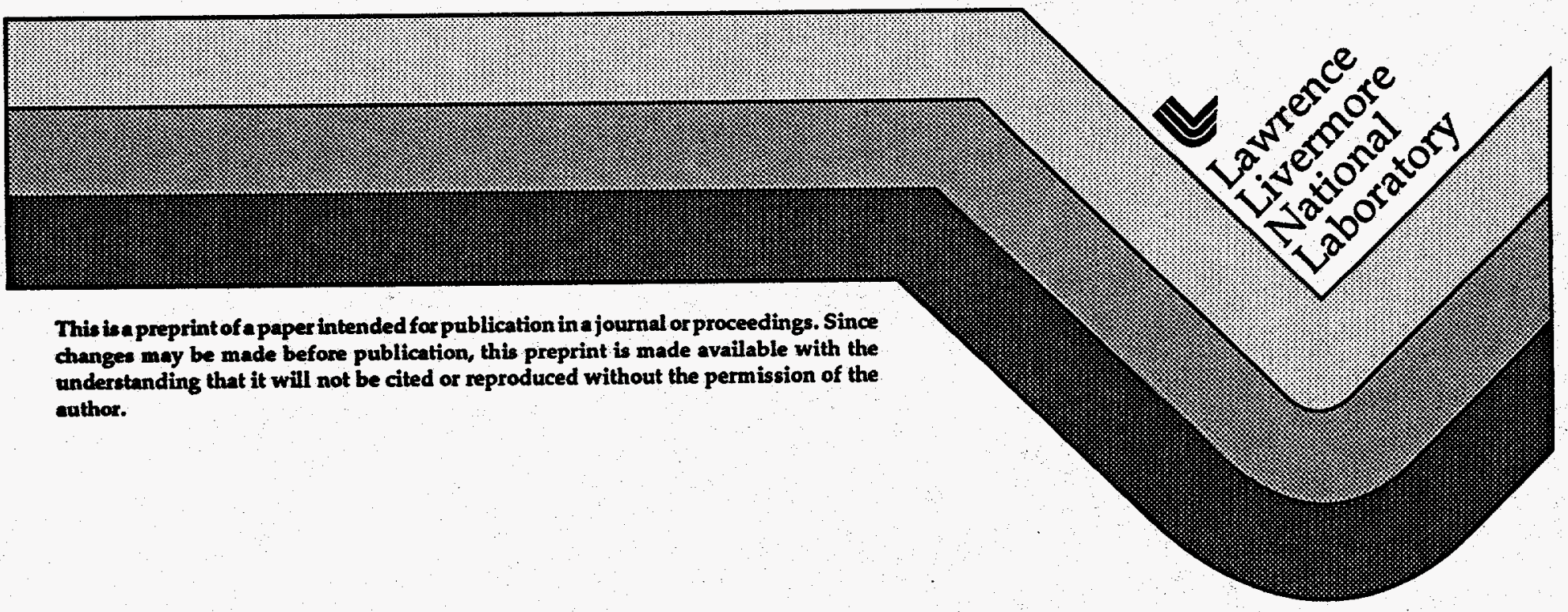




\section{DISCLAIMER}

This document was prepared as an account of work sponsored by an agency of the United States Government. Neither the United States Government nor any agency thereof, nor any of their employees, makes any warranty, expressed or implied, or assumes any legal liability or responsibility for the accuracy, completeness, or usefulness of any information, apparatus, product, or process disclosed, or represents that its use would not infringe privately owned rights. Reference herein to any specific commercial product, process, or service by trade name, trademark, manufacturer, or otherwise, does not necessarily constitute or imply its endorsement, recommendation, or favoring by the United States Government or any agency thereof. The views and opinions of authors expressed herein do not necessarily state or reflect those of the United States Government or any agency thereof. 


\section{DISCLAIMER}

Portions of this document may be illegible in electronic image products. Images are produced from the best available original document. 


\title{
Experimental Status of the AGS Relativistic Heavy Ion Program
}

\author{
T. Craig Sangster $\ddagger$ \\ Lawrence Livermore National Laboratory, Livermore, CA 94550
}

\begin{abstract}
The universal motivation for colliding large nuclei at relativistic energies is the expectation that a small volume of the primordial quark soup, generally referred to as the Quark-Gluon Plasma (QGP), can be created and studied. The QGP is formed via a phase transition caused by either the extreme baryon densities and/or the extreme temperatures achieved in the overlap zone of the two colliding nuclei. Experiments at the Brookhaven National Laboratory Alternating Gradient Synchrotron (AGS) using a beam of $\mathrm{Si}$ nuclei at $14.6 \mathrm{GeV}$ per nucleon on various nuclear targets have been completed. These same experiments are now actively searching for signatures of QGP formation using a beam of Au nuclei at $11.7 \mathrm{GeV}$ per nucleon. This paper briefly summarizes some of the key results from the Si beam program and the current status of the experimental Au beam program at the AGS.
\end{abstract}

\section{Introduction}

By colliding relativistic heavy ion beams with stationary nuclei, extremely dense baryonic matter is created in the laboratory. Estimates based on experimental observation and theoretical calculations indicate that densities between 4 and 9 times the groundstate matter density, $\rho_{0}$, should be achieved in small impact parameter collisions at energies of approximately 10 $\mathrm{GeV}$ per nucleon. Model predictions which explicitly include a nuclear matter equation-of-state (EOS) show considerable sensitivity to the EOS parameters at these densities. In addition, at these densities theorists predict that nuclear matter may undergo a transition from the normal bound states of quarks and gluons (hadronic matter) to a state in which the quarks and gluons are free to move within the high density volume. This primordial state is generally referred to as the Quark-Gluon Plasma (QGP) and has not existed since the Big Bang.

The experimental relativistic heavy ion program at the Brookhaven National Laboratory Alternating

† E-mail: sangster1@llnl.gov
Gradient Synchrotron (AGS) began in 1986, utilizing a 14.6 $\mathrm{GeV}$ per nucleon $\mathrm{Si}$ beam for a broad spectrum of measurements designed to study both detailed properties and gross features of $\mathrm{Si}+\mathrm{A}$ collisions. The original large experiments included E802, E810, E814 and E858. E802 measured particle spectra and the transverse energy of produced particles at mid-rapidity with extensive event characterization including total charged particle multiplicity and zero degree energy. E810 offered a $4 \pi$ examination of charge particle production using a series of time-projection chambers with limited single particle identification capabilities. E814 measured particle spectra forward of mid-rapidity and incorporated transverse energy measurements at both target and mid-rapidities. Finally, E858 consisted of a beam line spectrometer optimized to search at zero degrees for antinuclei as well as the standard suite of produced particles.

The current AGS experimental relativistic heavy ion program is based on measurements using an $11.7 \mathrm{GeV}$ per nucleon Au beam. All of the major experiments have upgraded to accomodate the higher charged particle multiplicities and most have added additional inclusive or event characterization measurements to study either

This work was performed under the auspices of the U.S. Department of Energy by Lawrence Livermore National Laboratory under contract No. W-7405-Eng-48. 


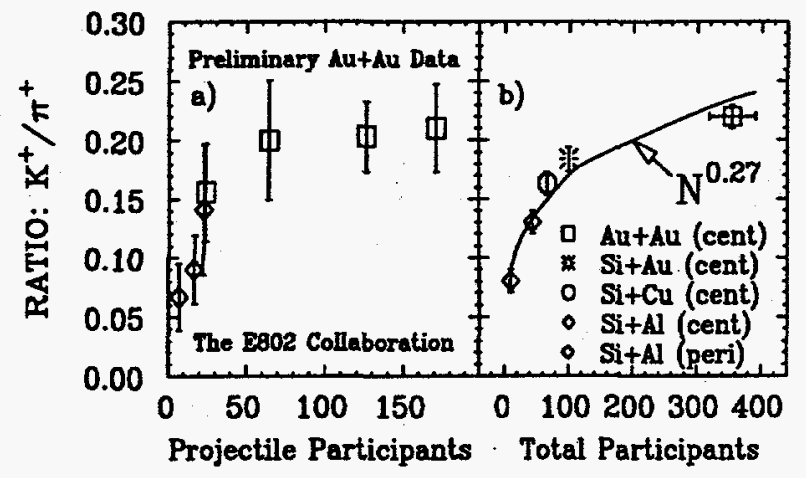

Figure 1. a) K/ $\pi$ ratio for the symetric systems $\mathrm{Si}+\mathrm{Al}$ and $\mathrm{Au}+\mathrm{Au}$ as a function of the number of projectile participants only. b) The $K / \pi$ ratio for central collisions of several systems as a function of the total number of participants (both target and projectile). The data in both panels are measurments by the E802 Collaboration.

new phenomena or extend the original Si beam coverage. Optimism is high that the much larger $\mathrm{Au}+\mathrm{A}$ systems will produce a considerable increase in both the spatial extent and the peak value of the maximum baryon densities achieved. Indeed, the experimental assault on the QGP phase boundary may be just beginning. The real challenge at the AGS is whether a subset of collisions in which some fraction of the system has crossed the deconfinement boundary can be identified using only hadronic probes (no AGS experiment utilizes leptonic probes). Since the phase boundary is reached via extreme densities at AGS energies, the key that unlocks the QGP may be a combination of both new and exotic probes and a systematic exploration of collective phenomena leading to an improved understanding of the nuclear EOS.

The remainder of the paper will give just a brief survey of some key $\mathrm{Si}$ beam results and, where available, mention the corresponding preliminary Au beam findings. The intent is to demonstrate both the scope of the anticipated Au beam measurements and the extent of our current understanding of relativistic heavy ion collisions.

\section{Recent Results}

At the AGS, the QGP would likely be created via high baryon densities rather than through extreme temperatures. Therefore, calorimeter based measurements of transverse energy, $E_{t}$, at mid-rapidity were among the first published $\mathrm{Si}$ beam results from experiments $\mathrm{E} 802$ and E814. These measurements [1, 2] showed a high degree of stopping in small impact parameter $\mathrm{Si}+\mathrm{A}$ collisions. In the context of relativistic heavy ion colli- sions, stopping means a lack of projectile energy in a forward cone, or effectively, the transfer of longitudinal beam momentum into transverse components. Stopping is generally equated with the creation of high baryon densities. Identical measurements with the Au+Au system [3] now show that the $E_{t}$ scales essentially with the number of projectile participants.

Due to the unavoidable rehadronization process, hadronic probes of QGP formation are not only difficult to measure but also difficult to interpret. Perhaps the most widely accepted potential hadronic signature is strangeness enhancement. Excess strangeness (via an enhancement in the $K / \pi$ ratio) was heralded early in the Si beam program but diligent efforts to understand the collision dynamics led to the realization that a number of non-QGP mechanisms (eg., rescattering of mesons and resonances) lead to higher than expected strangeness production. Figure 1 shows the $\mathrm{K}$ to $\pi$ ratios measured by the E802 series of experiments including preliminary data from the Au beam experiment, E866. Using the $K / \pi$ ratio to observe strangeness enhancement necessarily assumes that the $\pi$ yield represents a plasmaindependent benchmark of the temperature or density of the emitting system. Figure $1 \mathrm{a}$ shows the measured $\mathrm{K} / \pi$ ratio for the two symetric systems $\mathrm{Si}+\mathrm{Al}$ and $\mathrm{Au}+\mathrm{Au}$ as a function of the number of projectile participants. Here the ratio rises quickly with the number of projectile participants and then appears to saturate in the preliminary $\mathrm{Au}+\mathrm{Au}$ data indicating that the increased volume (and presumably, increased density) does not promote further strangeness production (as observed via the $K / \pi$ ratio). However, in central collisions of several different systems (Figure $1 \mathrm{~b}$ ), the measured $\mathrm{K} / \pi$ ratio continues to rise smoothly as a function of the total number of participating nucleons (both target and projectile). Clearly, the $K / \pi$ ratio is only one of the missing pieces in the QGP puzzle.

In general, collision models such as ARC [4] and RQMD [5] produce the observed $K / \pi$ ratios without invoking QGP formation, relying instead on rescattering mechanisms and the formation of resonance matter. Therefore, interest is high in measurments of multistrange, multi-quark objects such as strangelets [6] and $\Xi$ hyperons. Indeed experiment E810 has recently published results [7] on the production of the $\Xi^{-}$ relative to the singly strange $\Lambda$. Here it was found that the measured ratio of $N\left(\Xi^{-}\right)$to $N(\Lambda)$ is three times greater than predicted by a cascade model (AGSHIJET $+N^{*}$ ) suggesting an enhancement in the production of the multiply strange hyperons. Future experiments, discussed in the next section will focus heavily on these more exotic hadronic probes.

Identical particle correlations (HBT) have been studied extensively in the E802 series of experiments. These correlations can be used to estimate the space- 


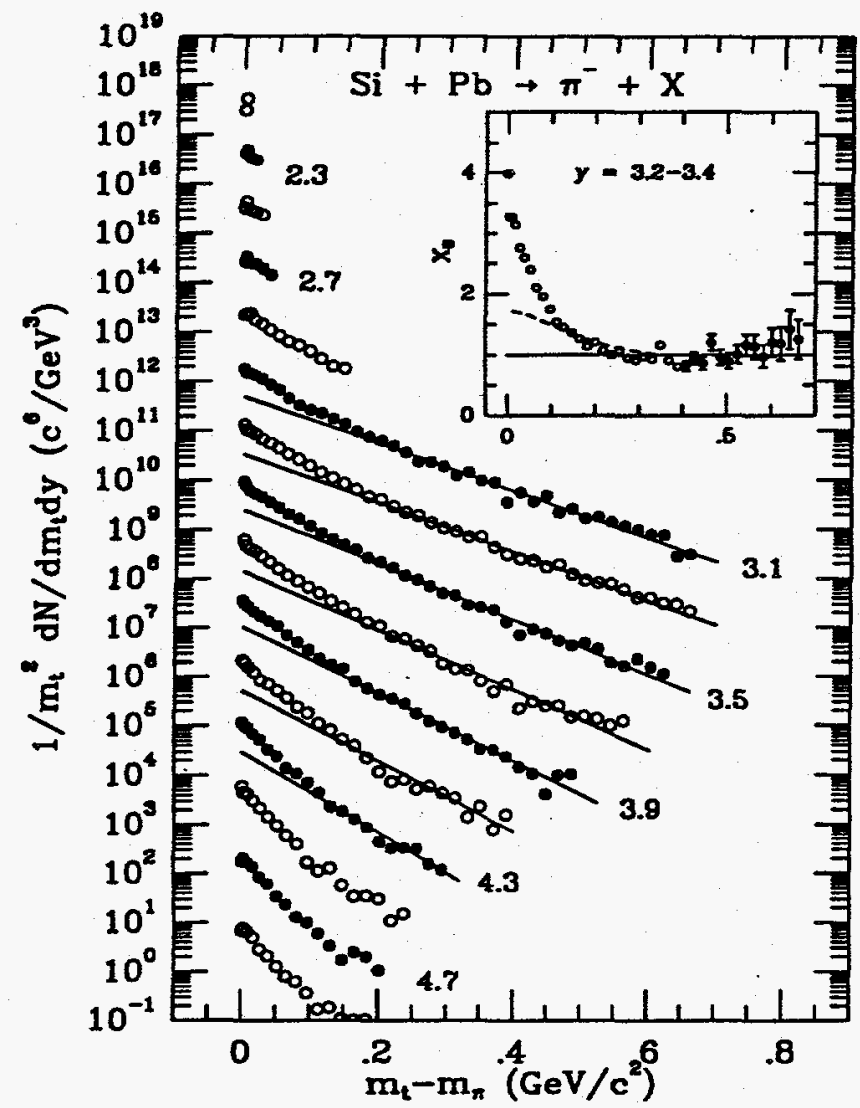

Figure 2. Experiment E814 $\pi^{-}$spectra for central $\left(\sigma / \sigma_{G E O}=2 \%\right) \mathrm{Si}+\mathrm{Pb}$ collisions and a variety of rapidity intervals. Starting with $y=4.7$, the data in each successively lower rapidity bin has been multiplied by increasing powers of 10. The lines through the data points are simple Boltzman fits to the region $m_{t}>300 \mathrm{MeV} / \mathrm{c}^{2}$.

time extent of the emitting source (the overlap region of the two colliding nuclei) which may be sensitive to the formation of the QGP. The $\mathrm{Si}$ beam results for both pions and kaons have been published [8]. The extracted RMS source radii are generally consistent with the nuclear radius of the Si projectile. Preliminary central $\mathrm{Au}+\mathrm{Au} \pi^{-} \pi^{-}$correlations indicate that the source radius again simply scales with the number of projectile partipants, ie, there is no apparent extended source which might suggest the formation of the QGP.

One of the most intriguing results from the Si beam program has been the observation of an enhancement in the transverse mass spectrum of pions at low $m_{t}$. The enhancement has been seen in data from both E810 and E814. Figure 2 shows $\pi^{-}$spectra for central $\mathrm{Si}+\mathrm{Pb}$ collisions from the E814 experiment [9] for a variety of rapidity intervals. As seen in the inset, the enhancement, relative to a Boltzman spectrum, reaches a factor of four in the lowest $m_{t}$ bin. A similar enhancement has been observed in the $\pi^{+}$spectra as well as with a much lighter $\mathrm{Al}$ target.
What makes this data so appealing is that the enhancement is likely caused by the decay of resonance matter. Both ARC and RQMD include contributions from $\Delta$ decays which preferentially produce pions with low transverse momenta in these rapidity intervals. Indeed, RQMD predicts that the density of purely resonance matter approaches that of normal nuclear matter at the peak of the total baryon density. The E814 collaboration has performed a straightforward calculation [10] which assumes that the pion enhancement is due entirely to the formation and decay of resonances. The results of this calculation indicate that approximately $30-40 \%$ of all nucleons in the participant zone are in the $\Delta$ resonance. In a purely thermal picture, this fraction can be explained by assuming a temperature of $140 \pm 20 \mathrm{MeV}$.

Figure 3 shows a composite of all particle species from central $\mathrm{Si}+\mathrm{Au}$ collisions measured by the $\mathrm{E802}$ collaboration (experiments E802 and E859). It is worth noting that a second level trigger implemented during E859 provided the necessary statistics to allow the $\phi$ and $\Lambda$ measurements as well as the superb $K^{-}$and $\bar{p}$ spectra. The addition of a forward spectrometer to the E802 apparatus will significantly extend the single particle coverage shown in the figure. A projectile hodoscope has also been implemented which will provide an event-by-event reaction plane for impact parameters less than $\approx 9$-fm. The E866 experiment will primarily focus on measurements of both the $\phi$, $\Lambda$ and resonance production as well as on single and multi-particle correlations with the event reaction plane (collective phenomena such as hydrodynamical flow and shadowing are likely measurements and should contribute significantly to our understanding of the nuclear EOS).

Finally, it is worth mentioning that excellent summaries of both the BNL and CERN relativistic heavy ion programs are available in the recent Quark Matter proceedings $[11,12]$ and the proceedings of the Heavy Ion Physics at the AGS (HIPAGS) workshops $[13,14]$.

\section{Future Experiments}

A number of new experiments are poised to begin making measurements at the AGS during the coming year. The first to take the floor will be experiment E864, a high sensitivity search for novel new forms of matter. E864 will focus primarily on the production of long-lived ( $\tau \geq 50 \mathrm{~ns}$ ) multi-strange quark matter (strangelets and the $\mathrm{H}^{0}$ dibaryon) and light antinuclei. The measurements will be made using an open geometry spectrometer with broad acceptance in both rapidity ( $\Delta y= \pm 0.5$ around mid-rapidity) and transverse momentum $\left(\mathrm{P}_{t} / \mathrm{Z} \leq 2 \mathrm{GeV} / \mathrm{c}\right)$. 
$14.6 \mathrm{~A} \cdot \mathrm{GeV} / \mathrm{c} \mathrm{Si}+\mathrm{Au}$ Central E802 + E859 Preliminary

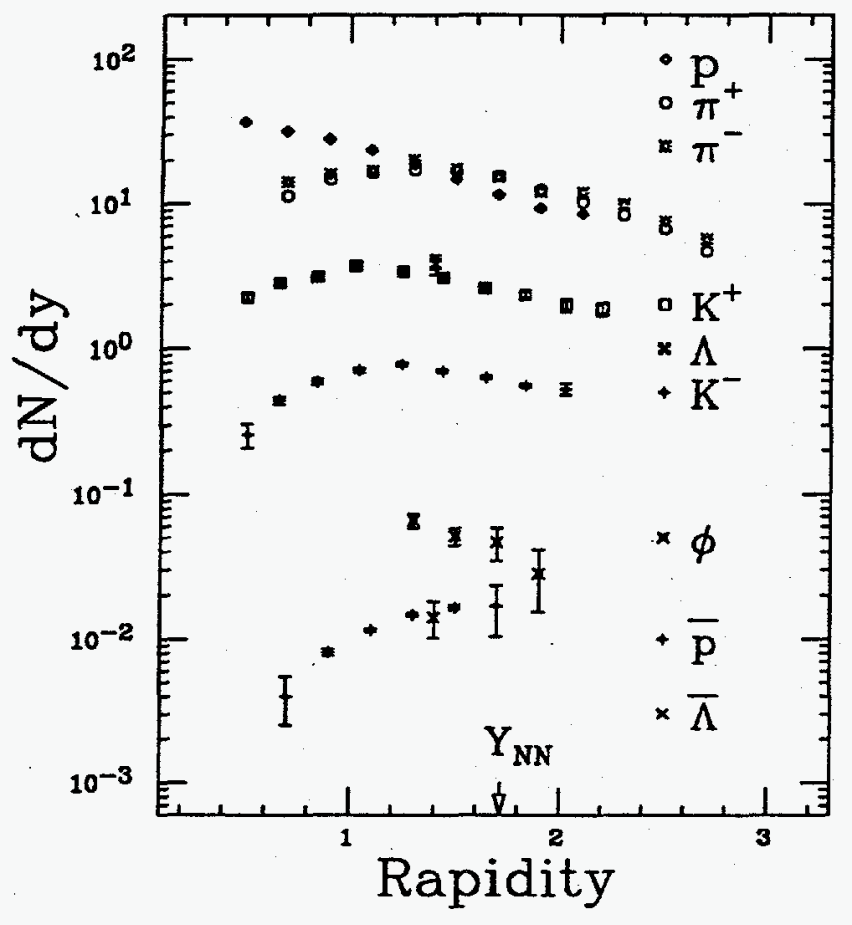

Figure 3. Composite of all measured particle species from experiments E802 and E859.

Experiment E896 will focus almost exclusively on a search for the $\mathrm{H}^{0}$ dibaryon via the decay topologies $\mathrm{H}^{0} \rightarrow \Sigma^{-} \mathrm{p}$ and $\mathrm{H}^{0} \rightarrow \Lambda \Lambda$. The experiment will be sensitive to shorter lifetimes than possible with the E864 apparatus and should be commissioned by 1995 . The multi-strange quark matter searches of E896 and E864 add an exciting new dimension to the experimental relativistic heavy ion program at the AGS and may be the best opportunity for identifying QGP signatures before the startup of the Relativistic Heavy Ion Collider (RHIC) in 1999.

Finally, E895 will carry out a systematic measurement of the energy and mass dependence of particle production, collective phenomena and multi-particle correlations in $\mathrm{Au}+\mathrm{A}$ reactions using the Lawrence Berkeley Laboratory EOS TPC, the MUSIC ion chamber and the EOS TOF wall. E895 will measure the four-momenta of light mass particles (including $\pi^{ \pm}, K^{ \pm}, K_{s}^{0}, \Lambda, \mathrm{n}, \mathrm{p}, \mathrm{d}$, and the isotopes of $\mathrm{He}, \mathrm{Li}$ and $\mathrm{Be}$ ), projectile fragments from $6 \leq Z \leq 79$ and anti-protons. It is expected that E895 will begin taking data during a lower energy Au beam run in 1995 .

\section{Summary}

To date there have been few surprises in the preliminary $\mathrm{Au}$ beam measurements. Quantities such as the total transverse energy and the identical particle correlation (HBT) radii appear to scale with the number of projectile participants. However, most of the Au beam data acquired to date has yet to be analyzed and, in this paper, the emphasis has been on what measurements are possible rather than on specific Au beam findings. The Si beam results suggest that the current set of experiments have the sensitivity to see new phenomena and model calculations indicate that, relative to $\mathrm{Si}+\mathrm{A}$ collisions, a significant increase in the $A u+A u$ central density should be expected. Furthermore, several new experiments are poised to look at new, exotic probes (the $\mathrm{H}^{0}$ dibaryon and strange quark matter) while current experiments are now beginning to probe new physics regimes (eg., collective phenomena) which promise a more complete understanding of the nuclear equation of state and the dynamics of hot, dense matter. Therefore, the outlook for the AGS relativistic heavy ion program is filled with expectations of discovery.

\section{References}

[1] T. Abbott et al., the E802 Collaboration, Phys. Lett B197 (1987) 285; Phys. Rev. C44 (1991), 1611; Phys. Rev C45 (1992) 2933.

[2] J. Barrette et al., the E814 Collaboration, Phys. Rev. Lett. 64 (1990) 1219.

[3] L. Ahle el al., the E802 Collaboration, submitted to Phys. Rev. Lett. (1994).

[4] Y. Pang et al., Phys. Rev. Lett. 68 (1992) 2743; T. J. Schlagel et al., Phys. Rev. Lett. 69 (1992) 3290.

[5] H. Sorge et al., Ann. Phys. (N.Y.) 192 (1989) 266; Nucl.Phys. A498 (1989) 567c.

[6] C. Griener, P Kock and H. Stoecker, Phys. Rev. Lett 58 (1987) 1825; C. Greiner, D. H. Rischke, H. Stoecker and P. Koch, Phys. Rev. D 38 (1988) 2797; C. Greiner and H. Stoecker, Phys. Rev. D 44 (1992) 3517.

[7] S. E. Eiseman et al., Phys. Lett. B325 (1994) 322.

[8] Y. Akiba et al., Phys. Rev. Lett. 70 (1993) 1057.

[9] T. K. Hemmick, Nucl. Phys. A566 (1994) 435 c.

[10] J. Stachel, Nucl. Phys. A566 (1994) 185c.

[11] Querk Matter' 91 , Proceedings of the Ninth International Conference on Ultra-Relativistic Nucleus-Nucleus Collisions, edited by T. C. Awes, F. E. Obenshain, F. Plasil, M. R. Strayer and C. Y. Wong, Gatlinburg, Tennessee, November 11-15, 1991, North Holland (1992); Nucl. Phys. A.544 (1992).

[12] Quark Matter '93, Proceedings of the Tenth International Conference on Ultra-Relativistic Nucleus-Nucleus Collisions, edited by E. Stenlund, H.-A. Gustafsson, A. Oskarsson and I. Otterlund, Borlange, Sweden, June 20-24, 1993, North Holland (1994); Nucl. Phys. A566 (1994).

[13] Proceedings of the Workshop on Heavy Ion Physics at the AGS, edited by $O$. Hansen, Brookhaven National Laboratory, Upton, New York, March 5-7, 1990, BNL-44911.

[14] HIPA GS '93, Proceedings of Heavy Ion Physics at the AGS, edited by G. S. F. Stephans, S. G. Steadman and W. L. Kehoe, MIT-LNS, Cambridge, Massachusetts, January 13-15, 1993, MITLNS-21 58. 